\title{
FUSÕES E AQUISIÇÕES DE EMPRESAS BRASILEIRAS: CRIAÇÃO DE VALOR E SINERGIAS OPERACIONAIS
}

\author{
MERGERS AND ACQUISITIONS IN BRAZILIAN COMPANIES: VALUE CREATION AND OPERATING SYNERGIES
}

\section{RESUMO}

O objetivo deste artigo foi avaliar se as fusões e aquisições de empresas brasileiras resultaram em sinergias operacionais e criaram valor para os acionistas. Além disso, buscou-se identificar a existência de correlação entre três medidas utilizadas de criação de valor, visando justificar o seu uso como proxies alternativas do desempenho empresarial. Assim, realizou-se um estudo descritivo que utizou os testes Wilcoxon Signed Rank Test e do Rank Test para analisar dados trimestrais de 72 processos de F\&A's ocorridos entre jan./96 e dez./04. As F\&A's estudadas resultaram em sinergias operacionais e na maximização do valor de mercado das empresas pesquisadas, e, aparentemente, da riqueza dos acionistas. Foram, ainda, encontradas correlações significativas entre as variáveis de criação de valor.

\section{Marcos Antônio de Camargos \\ Professor do Programa de Mestrado Acadêmico em Administração da Faculdade Novos Horizontes - MG, Brasil \\ marcos.camargos@unihorizontes.br}

\section{Francisco Vidal Barbosa}

Professor da Faculdade de Ciências Econômicas, Universidade Federal de Minas Gerais - MG, Brasil

fbarbosa@face.ufmg.br

Recebido em 16.07.2008. Aprovado em 17.10.2008

Avaliado pelo sistema double blind review

Editor Científico: Ricardo Ratner Rochman

\begin{abstract}
The objective of this article was to assess if mergers and acquisitions in Brazilian companies resulted in operating synergies and created value for shareholders. An attempt was also made to identify the existence of the correlation between three value creation measures used, with the aim of justifying their use as alternative proxies for corporate performance. A descriptive study was carried out that used the Wilcoxon Signed Rank Test and the Rank Test for analyzing the quarterly data of 72 Mergers \& Acquisitions processes that occurred between January 1996 and December 2004. The Mergers \& Acquisitions studied resulted in operating synergies and maximization of the market value of the companies surveyed and apparently of shareholder wealth. Significant correlations were also found between the value creation variables.
\end{abstract}

PALAVRAS-CHAVE Fusões e aquisições, criação de valor, sinergias operacionais, Wilcoxon Signed Rank Test, Rank Test. KEYWORDS Mergers and acquisitions, value creation, operating synergies, Wilcoxon Signed Rank Test, Rank Test. 


\section{INTRODUÇÃO}

Os processos de fusões e aquisições (F\&A's) são inerentes à concorrência capitalista e guardam estreita relação com as condições do mercado de capitais, mas principalmente com as grandes corporações. Nesse sentido, sua origem teórica se fundamenta nas diversas teorias que explicam a origem e o crescimento da firma. Trata-se de uma das atividades empresariais mais impactantes (para funcionários, empresa, socidade etc.), cujas consequências e desdobramentos empíricos ainda não estão totalmente explicados por teorias e regras no meio acadêmico. Ou seja, existe um vasto corpo de relatos e estudos empíricos que ainda carecem de um arcabouço teórico sistematizado.

Os processos de F\&A's integram a estratégia empresarial, e por meio deles as empresas conseguem expansão rápida, a conquista de novos mercados, maior racionalização produtiva, economias de escala, ativos complementares, entre outras coisas. Apesar disso, não devem ser encarados como uma solução simples para problemas internos ou ameaças mercadológicas, devido ao elevado grau de complexidade, risco e incerteza que permeiam essas operações, além da ausência de um consenso sobre ganhos (sinergias) e criação de valor.

Uma das obras mais citadas quando se trata de ganhos gerados por F\&A's é a de Jensen e Ruback (1983), que constataram que as F\&A's criam valor (econômico) para os acionistas a partir da oportunidade de utilizarem recursos específicos, que só poderiam ser obtidos por meio de F\&A's. Para Barney (1988) e Chatterjee (1986 e 1992), as F\&A's criam valor para o acionista somente quando as empresas conseguem duplicar as sinergias e o fluxo de caixa resultante da expectativa da união das empresas. Na perspectiva de McGee (1989), as F\&A's geralmente são benéficas para os acionistas de empresas adquiridas por rearranjarem os ativos corporativos de maneira mais eficiente, o que resulta no aumento do valor das ações para os acionistas e no aumento dos lucros para a empresa. Capron e Pistre (2002) constataram que as empresas adquirentes não obtinham retornos anormais quando recebiam apenas recursos das empresas adquiridas, mas poderiam ao contrário, obter esses ganhos quando transferiam seus próprios recursos para as empresas compradas.

Observa-se nos últimos anos que a economia brasileira tem apresentado um volume considerável de F\&A's, necessitando, portanto, de um melhor entendimento dos desdobramentos teóricos e práticos de tal atividade. É na identificação dos ganhos e na criação de valor proporcionadas pelos processos de F\&A's aos acionistas que reside a principal contribuição deste artigo, ao aumentar a compreensão de um tema controverso e relevante para a área acadêmica, ainda carente de teorias de sustentação. Além disso, apresenta também relevância para o meio empresarial, no sentido de identificar os benefícios (econômicos) gerados pelas principais combinações empresariais da economia brasileira em determinado período, mostrando para acionistas, gestores e sociedade os resultados da adoção dessa estratégia empresarial. Dessa maneira, espera-se que este trabalho contribua para preencher essa lacuna, aumentando as evidências empíricas sobre a atividade em questão, bem como contribuindo para a sistematização do seu arcabouço teórico.

Inserido nessa discussão, o objetivo deste artigo foi verificar se os processos de F\&A's de empresas brasileiras resultaram em sinergias operacionais e criaram valor para os acionistas. Além disso, investigou-se a correlação das três medidas de criação de valor utilizadas, no intuito de justificar o seu uso como proxies alternativas do desempenho / valor empresarial.

Na sequência, apresenta-se o quadro teórico de referência, seguido dos procedimentos metodológicos e da análise e discussão dos resultados nas seções 2,3 e 4, respectivamente. Encerra-se com as conclusões e considerações finais na seção 5 , seguidas das referências.

\section{REFERENCIAL TEÓRICO}

Segundo Cano (2002, p. 151),
Os processos de F\&A's são inerentes à concorrência capitalista. Acumulação de capital, inovações, ganhos de produtividade e acirramento da competição, levando a pressões pela eliminação de concorrentes ou pela abertura de novos mercados, são processos que marcaram a história do capitalismo desde o seu início. Tais fatores foram potencializados pelo surgimento do capital financeiro monopolista organizado na forma de sociedade anônima, pelo aumento da intervenção estatal na economia e pelo desenvolvimento do mercado bancário de capitais, o que impulsionou as F\&A's.

As atividades de F\&A's guardam, assim, relação estreita tanto com o surgimento, crescimento e "humor" do mercado de capitais quanto com o surgimento das grandes corporações, denominadas "corporações modernas" por Berle Jr. e Means (1932). Assim, sua origem teórica se fundamenta nas diversas teorias que explicam a origem e o crescimento da firma. 
Kloeckner (1994) salienta que a atividade de F\&A é um arcabouço teórico em construção, que ainda não está totalmente assimilado e sedimentado em uma teoria geral que lhe sirva de suporte, carecendo, portanto, tanto de estudos teóricos quanto empíricos. Para esses autores, o que existe é um conjunto de teorias que abordam algum aspecto do assunto, derivadas principalmente da teoria da firma e da agência.

No que se refere à questão da eficiência e dos ganhos (sinergias), Weston (1994) apresenta cinco teorias diferentes que explicam a atividade de F\&A, apresentadas no Quadro 1.

As ideias e consequências dessas teorias afetam tanto a distribuição da riqueza criada pelas firmas como também aspectos relacionados aos incentivos que os diversos stakeholders têm em investir e participar de atividades que resultem na criação de valor, no desenvolvimento das atividades das firmas. Na perspectiva da análise do desempenho e da criação de valor por uma empresa, Assaf Neto (2003) acrescenta que o uso de medidas centradas no valor traz a vantagem de enquadrar a administração da empresa aos objetivos dos acionistas, reduzindo os conflitos de agentes e oferecendo melhores práticas de governança corporativa.

\section{Criação de valor e sinergias em fusões e aquisições}

Cabe salientar aqui que a controvérsia existente na literatura sobre o resultado dos processos de F\&A's na perspectiva de criação de valor para o acionista aparentemente se prolongará indefinidamente por meio do embate teórico e das evidências empíricas favoráveis e desfavoráveis. As dificuldades de um consenso emergem dos seguintes fatores: 1) determinação de quão abrangente deve ser uma amostra para que se possam generalizar os resultados; 2) perspectiva de análise de curto, médio e longo prazo; 3 ) métricas a serem utilizadas (dados do mercado de capitais, variáveis de desempenho econômico ou financeiro?) e 4) como isolar os efeitos de um processo de F\&A sobre o desempenho de uma empresa, tendo em vista concomitância das demais estratégias adotadas. Certamente esses fatores são limitadores do escopo das pesquisas sobre a geração de riqueza para o acionista, mas não inviabilizadores das suas conclusões. Nesse sentido, os resultados de tais processos devem ser analisados sob a perspectiva de um mosaico de resultados, obtidos com diferentes dados e metodologias, que por si só não explicam o todo, mas em conjunto fornecem um panorama mais amplo desse debate e possibilitam um melhor entendimento do assunto.

Segundo Napier (1989), cerca de 30\% das F\&A's acabam em fracassos, e mais de $50 \%$ não alcançam completamente os objetivos pretendidos, sendo consideradas como insucessos. Salter e Weinhold (1979) atentam para a importância dos gestores na condução e na obtenção das sinergias, principalmente financeiras. Para eles, os gestores devem ter as habilidades necessárias para negociar com as várias situações e necessidades intrínsecas a um processo de $F \& A$ (e saber reconhecê-las), assim como ter conhecimento de vários setores, ter os recursos necessários e o acesso à fonte de financiamento adequada para sua execução.

Para Lang, Stulz, Walkling (1991), as empresas que ingressam em F\&A's sem ter como motivação principal a maximização de valor para o acionista caracterizam-se por preços baixos de ações em relação aos seus valores contábeis. Essas empresas compradoras são altamente lucrativas, mas por apresentarem baixos índices VM/VP, aparentemente, não se deve esperar que tenham um bom desempenho no futuro.

Os efeitos a longo prazo das F\&A's foram analisados por

\section{Quadro 1 - Hipóteses que discutem a eficiência e os ganhos em fusões e aquisições \\ HIPÓTESES \\ Hipótese da Eficiência \\ Hipótese da Informação \\ Hipótese da Agência \\ Hipótese do Poder de Mercado \\ Hipótese dos Impostos \\ ARGUMENTOS TEÓRICOS \\ Uma F\&A tanto pode melhorar o desempenho de uma empresa como produzir uma empresa mais eficiente, devido à obtenção de algum tipo de sinergia. \\ Efeito de curto prazo causado na cotação das ações das empresas envolvidas no mercado, que tenta incorporar ao preço o resultado ou o desdobramento da união. \\ As F\&A's podem ser um mecanismo para minimizar os problemas e custos de agência. \\ Se as economias esperadas pela F\&A não ocorrerem, 0 aumento na concentração foi motivado por ganhos de monopólio. \\ As F\&A's são motivadas por ganhos tributários.}

Fonte: Compilado de Weston (1994) pelos autores. 
Loughran e Vijh (1997), que concluíram: 1) os acionistas das empresas adquirentes obtêm retornos anormais negativos ou abaixo da média e, assim como a curto prazo, continuam tendo resultados insatisfatórios, com um retorno anormal médio de $-6,5 \% ; 2$ ) a forma de pagamento influencia a distribuição dos ganhos. Os acionistas de empresas adquirentes que pagam pela aquisição em dinheiro obtêm um retorno anormal médio de 18,5\%. Retorno que cai para -24,2\% quando a compra é paga com ações.

No que se refere às evidências sobre sinergias operacionais e financeiras em F\&A's utilizando dados contábeis, um trabalho ao mesmo tempo abrangente (mais de $5 \mathrm{mil}$ F\&As) e por isso questionado é o de Ravenscraft e Scherer (1987), que encontraram evidências de que elas não levaram a uma melhora de desempenho e por isso não criaram valor. Hasbrouck (1985), analisando o QT, constatou que as empresas-alvo frequentemente são empresas em decadência. Empresas bem administradas apresentavam QT elevado, em contrapartida com empresas mal administradas, nas quais esse indicador era baixo. Healy, Palepu e Ruback (1992) encontraram evidências de melhoras tanto nas vendas quanto nos lucros das empresas após a união. Andrade, Mitchell e Stafford (2001) constataram que as margens operacionais das empresas-alvo e adquirentes melhoraram aproximadamente $1 \%$ após a fusão.

Fazendo-se uma síntese dos principais resultados empíricos sobre a atividade de F\&A's, pode-se dizer que: 1) os acionistas de empresas adquiridas obtêm ganhos substanciais a curto prazo, quando a operação é bemsucedida, sendo os ganhos aparentemente maiores em ofertas de compras do que em fusões; 2) os acionistas das empresas adquirentes ganham relativamente pouco com as aquisições de controle, em média apenas 4\%; 3 ) a taxa de retorno para os acionistas de empresas que são alvo de operações malsucedidas de fusão é negativa e 4) a rentabilidade média geral para os acionistas em ofertas de compra e em fusões malsucedidas é igual (JENSEN e RUBACK, 1983; ASQUITH, 1983; LANG, STULZ e WALKLING; 1989; AGRAWAL, JAFFE e MANDELKER, 1992; GRAHAM, LEMMON e WOLF, 2002). Os resultados dos processos de F\&A's nos estudos acadêmicos são, assim, inconclusivos, e deixam em aberto a questão dos ganhos e benefícios para os acionistas, principalmente das empresas adquirentes.

\section{Sinergias e criação de valor em processos de F\&A's do mercado brasileiro}

Segundo Cano (2002), os processos de F\&A's ocorridos nos anos 1990 no Brasil foram fortemente influenciados: 1) pelo seu caráter institucionalizado, que fez com que boa parte das operações aqui ocorridas fosse apenas reflexo de F\&A's da arena internacional, ocasionando fusões e aquisições entre filiais; 2) pelo forte processo de reestruturação produtiva, retomada da entrada de fluxos de investimento direto estrangeiro (IDE) e expectativas favoráveis quanto à sustentabilidade do crescimento econômico, que desencadeou a entrada de novos concorrentes externos, boa parte via F\&A's e 3) pela reação das empresas já estabelecidas, nacionais ou estrangeiras, que também buscaram a estratégia de F\&A e a especialização no core business.

No contexto brasileiro, são poucos os trabalhos que analisaram a criação de valor e de sinergias operacionais em processos de F\&A's, alguns dos quais apresentados no Quadro 2.

Conforme se pode observar no Quadro 2, as pesquisas sobre desempenho financeiro, sinergias e criação de valor em processos de F\&A's com dados de empresas brasileiras ainda são muito incipientes. Os resultados das poucas existentes sinalizam para: 1) uma redução dos custos e despesas, gerando assim sinergias econômicas ou operacionais e 2) piora no desempenho financeiro pós-união.

O Quadro 3 apresenta a forma de cálculo e os pressupostos teóricos, bem como a fonte teórica e operacional das variáveis utilizadas neste estudo, como proxies da criação de valor e das sinergias em empresas que passaram por processos de F\&A's.

O QT é geralmente utilizado para medir o valor criado pela administração de uma empresa. A validade do QT para se mensurar a criação de valor para o acionista é justificada pelos trabalhos que a utilizaram para avaliar estratégias de diversificação, como: Wernerfelt e Montgomery (1988); Lang e Stulz (1994); Lloyd e Jahera (1994) e Steiner (1996). Quanto maior for o valor de mercado em relação ao seu valor patrimonial (VM/VP), maior é a perspectiva de geração de valor por parte da companhia. Ele também é conhecido como indicador do valor agregado da empresa, já que sinaliza a riqueza gerada por um empreendimento em termos da percepção do mercado relativa ao valor das suas ações. Geralmente as medidas QT, VM/VP e FV são utilizadas como proxies do valor de uma empresa, bem como do seu desempenho no mercado, em uma perspectiva de longo prazo.

No que se refere às variáveis de sinergias operacionais, segundo Silva (2006), muitas empresas têm estabelecido a remuneração de certos executivos a partir de metas de EBITDA, avaliando-os, assim, pela performance operacional, não computando o impacto dos investimentos em imobilizado. Segundo Damodaran (2004), a eficiência operacional de uma empresa determina sua margem 
operacional e, portanto, seu lucro operacional; empresas mais eficientes têm margens operacionais mais elevadas, quando outros fatores permanecem constantes, que empresas menos eficientes, que operam no mesmo setor. Assim, se uma empresa consegue aumentar sua margem operacional sobre os ativos existentes, ela vai gerar valor adicional.

\section{METODOLOGIA}

Esta pesquisa é do tipo descritiva por ter como objetivo principal a descrição das características de determinado fenômeno (F\&A) e o estabelecimento de relações entre variáveis. Trata-se também de uma pesquisa quantitativa, baseada em dados secundários, cujo objetivo foi identificar e descrever o impacto de determinado evento ( $F \& A$ ) sobre o desempenho operacional e das ações no mercado. Utilizou-se a técnica de pesquisa ex-post-facto, pois foram analisados fatos ocorridos no passado, após as variáveis terem interferido sobre o objeto de pesquisa, não sendo possível a interferência do pesquisador sobre as variáveis analisadas (COOPER e SCHINDLER, 2003).

A pesquisa é também do tipo cross-section, por ter um mesmo conjunto de empresas brasileiras de capital aberto analisadas em um período específico de tempo de nove anos. Foram analisados processos de F\&A's ocorridos após a implantação do plano de estabilização monetária (Plano Real), por se tratar de um período de relativa estabilidade monetária da economia brasileira, evitando assim possíveis vieses da análise de um período maior. As unidades de análise foram companhias de capital aberto, listadas na Bovespa, que passaram por processos de F\&A's após janeiro de 1996, e as unidades de observação foram os demonstrativos financeiros das empresas pesquisadas e a cotação das suas ações no mercado.

Foram objeto da pesquisa somente as companhias de capital aberto não financeiras que passaram por processos de fusões e aquisições entre janeiro (primeiro trimestre) de 1996 e dezembro (último trimestre) de 2004, cujas ações estavam listadas na Bovespa. Os dados contábeis e as cotações das ações foram obtidos do banco de dados Economática. No cálculo das variáveis foram utilizados dados trimestrais, obtidos de demonstrações financeiras não consolidadas, pois se buscou analisar o impacto da F\&A sobre a riqueza dos acionistas de determinada empresa e não do conglomerado empresarial a que ela pertence.

Os critérios de escolha da amostra foram: 1) identificação do dia da divulgação da F\&A ao mercado; 2) envolver um valor relevante (acima de R \$ 5 milhões); 3) envolver companhias não financeiras e 4) ter efetuado apenas um

Quadro 2 - Resumo dos trabalhos no mercado brasileiro

\begin{tabular}{|c|c|c|c|}
\hline AUTORES / ANO & AMOSTRA & $\begin{array}{l}\text { DADOS / } \\
\text { PERÍODO }\end{array}$ & CONCLUSÃO \\
\hline $\begin{array}{l}\text { Rocha, lootty, } \\
\text { Ferraz (2001) }\end{array}$ & $120 \mathrm{~F} \& \mathrm{~A}^{\prime} \mathrm{s}$ & $\begin{array}{l}\text { Contábeis } \\
\text { Anuais } \\
1990 / 1997\end{array}$ & $\begin{array}{l}\text { Não há diferenças significativas no desempenho econômico-financeiro } \\
\text { após a F\&A. }\end{array}$ \\
\hline $\begin{array}{l}\text { Matias e Pasin } \\
(2001)\end{array}$ & 22 F\&A's & $\begin{array}{l}\text { Contábeis } \\
\text { Anuais }\end{array}$ & $\begin{array}{l}\text { Grande parte das empresas apresentou reduções nos custos e despesas, } \\
\text { com conseqüente aumento na rentabilidade, comprovando a existência de } \\
\text { sinergias em F\&A's. }\end{array}$ \\
\hline Pasin (2002) & $15 \mathrm{~F} \& A^{\prime} S$ & $\begin{array}{l}\text { Contábeis } \\
\text { Anuais } \\
1996 / 1999\end{array}$ & $\begin{array}{l}\text { Os ganhos decorrentes de sinergias, integração entre empresas e melhor } \\
\text { gestão administrativa em processos de F\&A contribuíram para a redução de } \\
\text { despesas das empresas e a maior NCG contribuiu para a maior necessidade } \\
\text { de capital de terceiros ou próprios. }\end{array}$ \\
\hline $\begin{array}{l}\text { Camargos e } \\
\text { Barbosa (2005) }\end{array}$ & 22 F\&A's & $\begin{array}{l}\text { Contábeis } \\
\text { Anuais }\end{array}$ & $\begin{array}{l}\text { Identificaram uma piora na situação financeira das empresas analisadas, uma } \\
\text { melhora na situação econômica após a combinação, e que tais processos } \\
\text { geraram sinergias operacionais e gerenciais. }\end{array}$ \\
\hline $\begin{array}{l}\text { Gomes, Aidar e } \\
\text { Videira (2006) }\end{array}$ & $120 \mathrm{~F} \& \mathrm{~A}^{\prime} \mathrm{s}$ & $\begin{array}{l}\text { Contábeis } \\
\text { Anuais } \\
1998 \text { a } 2004\end{array}$ & $\begin{array}{l}\text { Existem claros sinais de aumento de lucratividade para toda a indústria, mas } \\
\text { com mais vigor nas participantes em F\&A's. }\end{array}$ \\
\hline
\end{tabular}




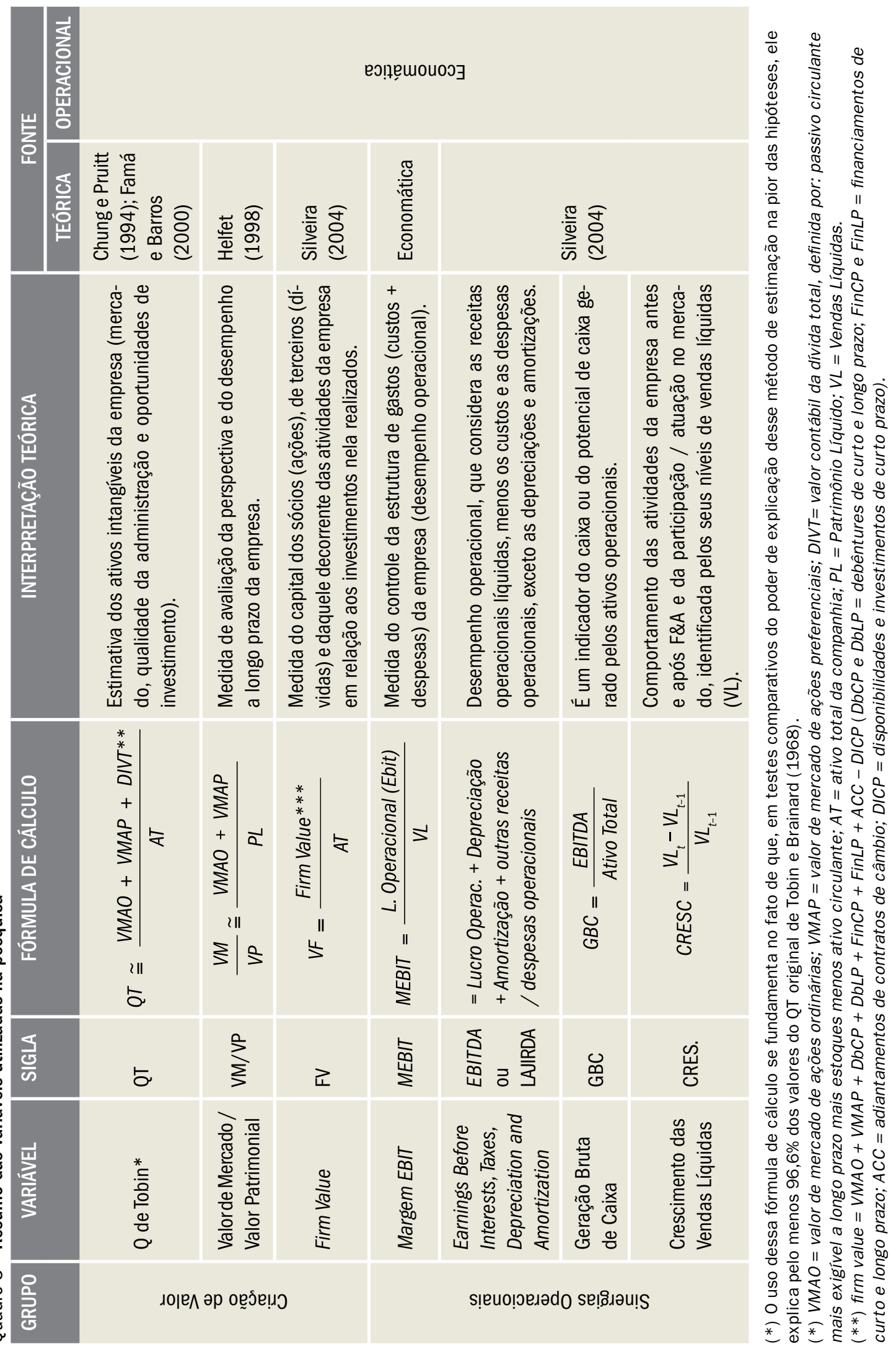


processo de F\&A no período de 24 meses (em caso de mais de um, foi selecionado o de maior valor para a empresa, ou aquele considerado mais estratégico, como, por exemplo, F\&A's que significassem a entrada ou a ampliação da participação em mercados estrangeiros).

Foram analisados dados de setenta e seis (76) empresas, que participaram de setenta e dois (72) processos de F\&A's, que envolveram sessenta e quatro (64) companhias diferentes, distribuídas em: a) quarenta e nove (49) empresas compradoras / adquirentes; b) treze (13) empresas compradas / alvo; c) duas (2) que se processaram como fusões nas quais não se observou a figura nem de adquirente nem de adquirida, nos cinco (5) processos de fusão analisados.

A Tabela 1 apresenta a distribuição dos processos de F\&A's analisados no período estudado.

Na ausência de uma metodologia consolidada, optou-se por fazer uma análise (comparação) da média das variáveis de cada empresa nos períodos anterior e posterior (dois anos) à divulgação da F\&A. Essa metodologia é a mesma adotada por Megginson, Nash e Randenborgh (1994) e Pinheiro (1996) para empresas privatizadas, e Rocha, Iootty e Ferraz (2001) para empresas que empreenderam processos de F\&A's. Trata-se especificamente da comparação da média de cada variável de todas as empresas nos oito trimestres anteriores à união empresarial com os oito trimestres posteriores, utilizando-se como ferramentas estatísticas o Wilcoxon Signed Rank Test e o Rank Test.

$$
\sum_{t=-8}^{-1} \frac{\left(\sum_{i=1}^{n} \operatorname{Var}_{X, t}\right)}{n} \Leftrightarrow \sum_{t=+1}^{+8} \frac{\left(\sum_{i=1}^{n} \operatorname{Var}_{X, t}\right)}{n}
$$

sendo:

$t=$ trimestres relativos analisados $(-8 a-1$ anteriores $e+1 a$ +8 posteriores à união); $n=$ número total de empresas que tiveram a variável calculada e analisada; $\operatorname{Var} X=$ variáveis analisadas; $i=$ empresa analisada.

Como a economia mundial e brasileira apresentou um resultado distinto no período estudado, optou-se por di- vidi-lo em dois, baseado no comportamento do Ibovespa (a maioria das empresas da amostra compõe esse índice). O mês de dez./99 foi tomado como divisor, porque nele o Ibovespa ultrapassou pela primeira vez a marca dos 15 mil pontos, apresentando, também, uma média distinta. No Período 1 - P1 - (jan./94 até dez./99), essa média foi de 7.149 pontos, enquanto no Período 2 - P2 - (jan./00 a dez./06) foi de 20.634 pontos. Presumia-se com essa divisão que possivelmente as F\&A's ocorridas no segundo apresentariam resultados mais contundentes de valorização acionária (criação de valor) e de melhora no desempenho operacional, fornecendo assim indícios de que o comportamento da economia brasileira possa ter influenciado o resultado das F\&A's estudadas. Além disso, dividiu-se a amostra entre empresas adquirentes, adquiridas e fusionadas, visando identificar possíveis diferenças nos seus resultados. Cabe ressaltar, entretanto, que o foco principal de análise é a amostra com todas as empresas $(n=76)$.

\section{Variáveis utilizadas e hipóteses testadas}

Conforme apresentado no Quadro 3, foram utilizadas três variáveis para mensurar a criação de valor: $\mathrm{Q}$ de Tobin (QT), Valor de Mercado / Valor Patrimonial (VM/ VP) e Valor da Firma (FV). No cálculo do valor de mercado das ações ON e PN, foram utilizadas cotações não ajustadas a proventos, multiplicadas pelo total de cada ação, excluindo-se as ações em tesouraria. Os dados contábeis foram referentes ao respectivo trimestre, e a cotação das ações corresponde ao preço de fechamento do último dia de negociação de cada trimestre. Em caso de ausência de informação para as ações ordinárias, foi utilizada uma aproximação pela cotação da ação preferencial, conforme Silveira (2004), que apresenta maior liquidez em relação àquelas no mercado brasileiro.

As sinergias operacionais foram analisadas por meio de quatro variáveis, Margem EBIT (MEBIT), EBITDA (LAJIRDA), Geração Bruta de Caixa (GBC) e Crescimento da Receita Líquida (CRES.). Para as variáveis EBITDA e GBC foram utilizados dados anuais, ou seja, dois anos anteriores e dois posteriores.

As hipóteses teóricas testadas neste estudo foram:

Tabela 1 - Distribuição dos processos de F\&A's no período

\begin{tabular}{|l|c|c|c|c|c|c|c|c|c|c|}
\hline \multicolumn{1}{|c|}{ ANO } & 1996 & 1997 & 1998 & 1999 & 2000 & 2001 & 2002 & 2003 & 2004 & TOTAL \\
\hline $\mathrm{N}^{0}$ F\&A's & 13 & 8 & 4 & 8 & 9 & 1 & 7 & 5 & 17 & 72 \\
\hline $\mathrm{N}^{0}$ Empresas & 15 & 8 & 5 & 8 & 9 & 1 & 7 & 5 & 18 & 76 \\
\hline
\end{tabular}


$\mathrm{H}_{1}$ : A média das variáveis de criação de valor das empresas que passaram por processos de fusão e aquisição não sofreu mudanças significativas após a união.

$\mathrm{H}_{2}$ : A média das variáveis de sinergias operacionais das empresas brasileiras que passaram por processos de fusão e aquisição não sofreu mudanças significativas após a união.

$\mathrm{H}_{3}$ : $\mathrm{O}$ resultado das variáveis analisadas de empresas que passaram por processos de fusão e aquisição não apresenta diferenças quando se observam subamostras de empresas adquirentes, adquiridas e de fusões.

$\mathrm{H}_{4}$ : O resultado das variáveis analisadas de empresas que passaram por processos de fusão e aquisição no Período 1 não foi diferente daquele observado nas empresas no Período 2.

$\mathrm{H}_{5}$ : Não existe correlação com significância estatística entre as variáveis QT, VM/VP e FV.

\section{Tratamento estatístico}

Visando identificar o impacto das F\&A's estudadas no que se refere à criação de valor e sinergias operacionais nas empresas da amostra, foram feitos testes de diferença de médias de cada uma das variáveis descritas na seção anterior. Após se constatar que poucas variáveis apresentavam distribuição normal, optou-se por utilizar o Wilcoxon Signed Rank Test e o Rank Test (Teste do Sinal). O segundo mede a proporção de empresas que apresentaram mudanças no sentido esperado e o primeiro ajusta essa medida levando em conta a magnitude da mudança (PINHEIRO, 1996).

O Wilcoxon Signed Rank Test e o Rank Test são testes não paramétricos que testam a hipótese de um determinado evento ( $F \& A$, no caso deste artigo) não ter efeito após a sua ocorrência, usando, para isso, pares de variáveis casadas (BICKEL e DOKSUM, 1977). Por exemplo, considere-se a variável QT. Para a amostra de 76 empresas, é possível supor, formalmente, 76 pares independentes de variáveis aleatórias $\left(X_{1 j}, X_{2 j}\right)$, sendo $j=1,2 \ldots ., n \operatorname{com} n$ = número de pares de variáveis analisadas (i.e., número de empresas); $X_{1}=$ média do QT da empresa nos dois anos anteriores à operação, e $X_{2}=$ média do QT da empresa nos dois anos posteriores à operação. Nesse caso, para cada par $\left(X_{1 j}, X_{2 j}\right)$, o impacto (resultado) da realização da(s) operação(ões) de F\&A's pode ser medido por $R j=X_{1 j}-X_{2 j}$, para $j=1,2 \ldots ., n$. A hipótese nula, de que a realização de $F \& A$ não cria valor para o acionista e não afeta o desempenho financeiro da empresa, corresponde à hipótese de que $R_{j}$ tem uma distribuição simétrica em relação a zero. Em ambos os testes foi considerado um nível de significância de 5\% ( $p$-value <0,05).

$\mathrm{Na}$ análise da correlação entre as três variáveis de criação de valor utilizou-se também o Coeficiente de
Correlação de Pearson $(r)$. Os testes estatísticos foram efetuados utilizando-se o Statistical Package for the Social Sciences (SPSS), versão 13.0.

\section{RESULTADOS}

As Tabelas 2 e 4 resumem os resultados obtidos para cada um dos grupos de variáveis, a partir da amostra e das subamostras analisadas. Mais precisamente, são apresentados: a magnitude da variação entre os valores das médias das variáveis antes e depois do "trimestre da F\&A" (variação na média); a proporção das empresas que apresentaram variação positiva na referida variável (crescimento na variável); o resultado à luz da teoria das finanças e os níveis de significância relativos ao Wilcoxon Signed Rank Test e ao Rank Test.

Analisando-se os dados da amostra de todas as empresas ( $n=76)$, encontraram-se evidências (com significância estatística) da criação de valor nas três variáveis, QT, $\mathrm{VM} / \mathrm{VP}$ e $\mathrm{FV}$, pois $65,7 \%, 59,1 \%$ e $63,7 \%$ das empresas apresentaram elevação média de 27,6\%, 84,8\% e 15,1\%, respectivamente, após a F\&A. Assim, rejeitou-se $\mathrm{H}_{1}$. Ou seja, a análise conjunta dessas três variáveis permite concluir que os processos de F\&A's estudados resultaram na maximização do valor de mercado das empresas pesquisadas e, consequentemente, da riqueza dos acionistas. Entretanto, constatou-se uma piora na variável FV somente para a amostra de empresa adquirida, mas sem significância estatística. Nas demais subamostras observou-se uma melhora com significância estatística para todas as empresas.

Já quanto às empresas adquirentes $(\mathrm{n}=63), 67,7 \%$, $62,1 \%$ e $65,7 \%$ delas aumentaram suas médias, no período posterior à $\mathrm{F} \& \mathrm{~A}$, em $25,9 \%, 52,6 \%$ e $24,9 \%$, respectivamente. Entretanto, foram as empresas do P2 que apresentaram as evidências mais significativas de melhora das médias dessas variáveis: 76,3\%, 64,3\% e 73,8\% delas com elevação da média em 32,5\%, 64,3\% e 24,5\%, respectivamente (todas com significância de 1\%). Cabe aqui a ressalva de que possivelmente essa criação de valor pode não ser advinda da estratégia de F\&A, tendo em vista que se trata de um período, principalmente a partir de 2000, no qual ocorreram melhorias dos fundamentos microeconômicos do país, impulsionadas em parte pelo crescimento do valor das commodities, produtos básicos de exportação e de faturamento de boa parte das grandes empresas brasileiras, inclusive da amostra estudada.

Em síntese, as F\&A's resultaram na maximização do valor de mercado das empresas pesquisadas e, aparente- 
mente, da riqueza dos acionistas, no período analisado, principalmente para as empresas adquirentes e para as F\&A's que ocorreram no $\mathrm{P} 2$, rejeitando-se, portanto, $\mathrm{H}_{1}$. As três variáveis sinalizadoras da criação de valor, QT, VM/ $\mathrm{VP}$ e FV, apresentaram mudanças significativas e positivas, confirmando a criação de valor pelas empresas estudadas, nas três subamostras analisadas. Além disso, $\mathrm{H}_{4}$ também foi refutada, pois enquanto as F\&A's do P1 apresentaram apenas indícios de melhora (sem significância estatística), as F\&A's ocorridas no P2 apresentaram resultados bem mais contundentes da criação de valor, com evidências (significância estatística) de melhoras nas três variáveis.

$\mathrm{Na}$ análise da existência de correlação entre as variáveis de criação de valor QT, VM/VP e FV (Tabela 3), os resultados levaram à rejeição de $\mathrm{H}_{5}$, pois, conforme se pode observar, há correlação significante ao nível de 1\% quando se compara cada uma das três variáveis nos respectivos períodos, anteriores e posteriores. Essa constatação reforça o pressuposto de que essas três variáveis podem ser utilizadas como proxies alternativas umas das outras em estudos acadêmicos, uma vez que apresentaram comportamento semelhante. Há que se salientar, entretanto, que foi constatada uma proximidade de comportamento maior entre as variáveis QT e VM/VP (90,6\% antes e $84,6 \%$ depois da F\&A), significando dizer que a variação da primeira se aproxima em mais de $84 \%$ da variação da segunda. Variação esta bem superior à verificada com as variáveis QT e FV (58,8\% antes e 70,6\% depois da F\&A) e de VM/VP e FV (54,1\% e 28,0\%), sinalizando que a variável VM/VP pode ser utilizada como proxy do QT, que o FV deve sê-lo com ressalvas (baixa correlação), enquanto as variáveis VM/VP e FV não devem ser utilizadas como proxy uma da outra, tendo em vista a baixa correlação apresentadas entre ambas.

$\mathrm{Na}$ análise das sinergias operacionais geradas pelas F\&A's estudadas (Tabela 4), de forma geral, os resultados obtidos indicam uma melhora no desempenho das empresas após a F\&A (exceto para a variável Cres.), o que implica dizer que para três das quatro variáveis de sinergias operacionais consideradas no estudo rejeitou-se $\mathrm{H}_{2}$.

A Margem EBIT melhorou em todas as subamostras analisadas e com significância estatística (exceto para as F\&A's ocorridas no P2), com destaque para as empresas adquiridas, F\&A's do P1 e fusões, cuja média aumentou $38,3 \%, 28,9 \%$ e $13,4 \%$, respectivamente, com um percentual acima de $63 \%$ das empresas melhorando suas margens. Resultado semelhante foi observado na variável EBITDA, que melhorou em todas as subamostras pesquisadas, mas com significância estatística apenas para as F\&A's ocorridas no P1, todas as empresas da amostra, F\&A's do
P2 e empresas adquirentes, cujas médias aumentaram $58,4 \%, 49,3 \%, 47,9 \%$ e $47,1 \%$, respectivamente, com um percentual acima de $65 \%$ das empresas melhorando o lucro obtido nas suas operações.

A variável GBC apresentou resultados sem significância estatística e contraditórios, ao melhorar em três das subamostras analisadas (todas as empresas, empresas adquiridas e F\&A's do P1), e piorar nas outras três (empresas adquirentes, fusões e F\&A's do P2). Chegou-se, portanto, a resultados inconclusivos para esta variável. Uma possível explicação para isso é que o aumento do AT, característico de operações de F\&A, tenha compensado o aumento observado na variável EBITDA.

A variável Cresc. apresentou evidências contrárias às variáveis ligadas ao lucro operacional, ao piorar em quatro das subamostras ( $F \& A$ 's do P1, todas as empresas, empresas adquirentes e $F \& A$ 's do P2), cujas médias reduziram $51,2 \%, 47,3 \%, 42,3 \%$ e $39,7 \%$, respectivamente, com um percentual acima de $54 \%$ das empresas passando a ter um volume menor de vendas após a F\&A. Uma razoável interpretação é entender essa redução como resultado de ajustes no market share e redefinição nos segmentos de atuação após a F\&A. A evidência da redução na média da variável Cresc., associada à evidência (MEBIT e EBITDA) e aos indícios (GBC) de melhora de variáveis sinalizadoras da margem de lucro e do lucro operacional, reforça a ideia do aumento da eficiência operacional. Ou seja, reduziram-se as receitas, mas aumentaram-se os lucros. O que permite concluir que as F\&A's estudadas resultaram em sinergias operacionais para as empresas após a combinação, rejeitando-se, portanto, $\mathrm{H}_{2}$.

$\mathrm{Na}$ comparação entre $\mathrm{P} 1$ e P2, considerando as variáveis de sinergias operacionais, rejeitou-se também $\mathrm{H}_{4}$, mas com resultados contrários aos esperados, com a F\&A's do Pl apresentando melhoras em três das variáveis (MEBIT, EBITDA e GBC), com significância estatística nas duas primeiras. Enquanto as F\&A's ocorridas no $\mathrm{P} 2$ apresentaram melhoras apenas nas duas primeiras variáveis e com significância estatística apenas para a variável EBITDA. As F\&A's do P1 apresentaram um melhor desempenho do que as F\&A's do P2. Uma possível explicação para isso seria a necessidade de adaptação à nova realidade econômica, que exigiu maior eficiência operacional das empresas, ao reduzir as possibilidades de ganhos não operacionais no mercado financeiro, levando-as a se especializarem no seu core business (CANO, 2002).

Por fim, $\mathrm{H}_{3}$ também foi rejeitada, uma vez que as primeiras apresentaram evidências de maior potencial de criação de valor após a união, quando comparadas com as demais. Resultado semelhante se observa quando as em- 
presas adquirentes apresentaram evidências de melhora nas duas variáveis de desempenho operacional (MEBIT, EBITDA), enquanto as empresas adquiridas também o tiveram, mas somente na última variável. Ou seja, empresas adquirentes apresentaram um maior potencial de criação de valor e de gerar sinergias operacionais, quando comparadas com empresas adquiridas e fusionadas.

Por fim, há que se destacar o papel desempenhado pelos executivos nos processos de F\&A's. Papel esse que vai desde a identificação de possíveis empresas a serem adquiridas, passando pela análise minuciosa das variáveis e condições envolvidas na combinação (due deligence), indo até a gestão do processo de combinação em si. Qualquer uma dessas etapas envolve uma análise criteriosa e precisa por parte dos gestores, tendo em vista que erros, intencionais ou não, podem ocasionar o insucesso de tais proces- sos ou a destruição de riqueza dos acionistas. Outro fator que pode influenciar negativamente os executivos nessas etapas é o conflito de interesses existente nas grandes corporações entre os objetivos deles e o dos acionistas (teoria da agência). Além disso, os executivos de empresas que passam por F\&A's são os responsáveis diretos pelo seu sucesso ou fracasso, pois têm atuação decisiva na condução do processo, estabelecendo as regras, as metas, os objetivos e os resultados esperados do processo.

\section{CONSIDERAÇÕES FINAIS E CONCLUSÃO}

Analisou-se neste artigo se os processos de F\&A's de empresas brasileiras resultaram em sinergias operacionais e criaram valor para os acionistas. Além disso, investigou-se

Tabela 2 - Resumo do resultado do teste de comparação de médias para variáveis de criação de valor

\begin{tabular}{|c|c|c|c|c|c|c|c|c|c|c|}
\hline $\mathrm{N}^{0}$ & AMOSTRA & VARIÁVEL & $\begin{array}{c}\mathrm{N}^{\circ} \\
\text { OBS. }\end{array}$ & $\begin{array}{l}\text { MÉDIA } \\
\text { ANTES }\end{array}$ & $\begin{array}{l}\text { MÉDIA } \\
\text { DEPOIS }\end{array}$ & $\begin{array}{l}\text { VARIAÇÃO } \\
\text { NA MÉDIA }\end{array}$ & $\begin{array}{l}\text { CRESCIMENTO } \\
\text { NA VARIÁVEL }\end{array}$ & RESULTADO & $\begin{array}{l}\text { NIVEL SIG- } \\
\text { NIFICÂNCIA } \\
\text { - TESTE } \\
\text { WILCOXON - }\end{array}$ & $\begin{array}{c}\text { NIIVEL SIG- } \\
\text { NIFICÂNCIA } \\
\text { - TESTE DO } \\
\text { SINAL- }\end{array}$ \\
\hline 1 & \multirow{3}{*}{$\begin{array}{l}\text { Todas as } \\
\text { Empresas } \\
(\mathrm{N}=76)\end{array}$} & QT & 592 & 0,997 & 1,272 & $27,6 \%$ & $65,7 \%$ & melhorou & 0,000 & 0,000 \\
\hline 2 & & VM/VP & 592 & 1,450 & 2,680 & $84,8 \%$ & $59,1 \%$ & melhorou & 0,000 & 0,000 \\
\hline 3 & & FV & 592 & 1,075 & 1,238 & $15,1 \%$ & $63,7 \%$ & melhorou & 0,000 & 0,000 \\
\hline 1 & \multirow{3}{*}{$\begin{array}{l}\text { Adquirentes } \\
(\mathrm{N}=63)\end{array}$} & QT & 504 & 0,993 & 1,250 & $25,9 \%$ & $67,7 \%$ & melhorou & 0,000 & 0,000 \\
\hline 2 & & VM/VP & 504 & 1,460 & 2,229 & $52,6 \%$ & $62,1 \%$ & melhorou & 0,000 & 0,000 \\
\hline 3 & & FV & 504 & 1,037 & 1,295 & $24,9 \%$ & $65,7 \%$ & melhorou & 0,000 & 0,000 \\
\hline 1 & \multirow{3}{*}{$\begin{array}{l}\text { Adquiridas } \\
(\mathrm{N}=13)\end{array}$} & QT & 96 & 1,232 & 1,728 & $40,2 \%$ & $56,3 \%$ & melhorou & 0,265 & 0,262 \\
\hline 2 & & VM/VP & 96 & 1,799 & 5,821 & $223,5 \%$ & $44,8 \%$ & melhorou & 0,389 & 0,358 \\
\hline 3 & & FV & 96 & 1,476 & 1,345 & $-8,9 \%$ & $54,2 \%$ & piorou & 0,419 & 0,475 \\
\hline 1 & \multirow{3}{*}{$\begin{array}{l}\text { Fusões } \\
(N=6)\end{array}$} & QT & 40 & 1,589 & 1,679 & $5,7 \%$ & $60,0 \%$ & melhorou & 0,485 & 0,268 \\
\hline 2 & & VM/VP & 40 & 2,148 & 2,033 & $-5,3 \%$ & $37,5 \%$ & piorou & 0,412 & 0,155 \\
\hline 3 & & $\mathrm{FV}$ & 40 & 1,429 & 1,600 & $11,9 \%$ & $50,0 \%$ & melhorou & 0,591 & 1,000 \\
\hline 1 & \multirow{3}{*}{$\begin{array}{l}\text { Período } 1 \\
(\mathrm{~N}=36)\end{array}$} & QT & 272 & 0,806 & 0,961 & $19,3 \%$ & $53,3 \%$ & melhorou & 0,751 & 0,303 \\
\hline 2 & & VM/VP & 272 & 1,023 & 2,328 & $127,6 \%$ & $43,4 \%$ & melhorou & 0,393 & 0,034 \\
\hline 3 & & FV & 272 & 0,816 & 0,797 & $19,3 \%$ & $53,3 \%$ & melhorou & 0,751 & 0,303 \\
\hline 1 & \multirow{3}{*}{$\begin{array}{l}\text { Período } 2 \\
(\mathrm{~N}=40)\end{array}$} & QT & 320 & 1,160 & 1,537 & $32,5 \%$ & $76,3 \%$ & melhorou & 0,000 & 0,000 \\
\hline 2 & & VM/VP & 320 & 1,812 & 2,979 & $64,3 \%$ & $72,5 \%$ & melhorou & 0,000 & 0,000 \\
\hline 3 & & FV & 320 & 1,295 & 1,612 & $24,5 \%$ & $73,8 \%$ & melhorou & 0,000 & 0,000 \\
\hline
\end{tabular}


a correlação das três medidas de criação de valor, visando justificar o seu uso como proxies alternativas do desempenho da empresa. Foram analisados dados econômicos, contábeis e de mercado de uma amostra de 72 processos de F\&A's de empresas brasileiras ocorridos entre janeiro de 1996 e dezembro de 2004.

Os principais resultados encontrados nesta pesquisa foram: 1) mudanças significativas e positivas na média das três variáveis sinalizadoras de criação de valor (QT, FV e VM/VP), resultando, assim, na maximização do valor de mercado das empresas pesquisadas e, aparentemente, da riqueza dos acionistas (rejeitou-se $\mathrm{H}_{1}$ ); 2) as F\&A's estudadas resultaram em sinergias operacionais para as empresas após a combinação (rejeitou-se $\mathrm{H}_{2}$ ); 3 ) as empresas adquirentes apresentaram maior potencial de criação de valor e de gerar sinergias operacionais quando comparadas com empresas adquiridas e fusionadas (rejeitou-se $\mathrm{H}_{3}$ ); 4) as F\&A's do P2 apresentaram resultados bem mais contundentes da criação de valor, enquanto, para as variáveis de sinergias operacionais, os resultados foram contrários aos esperados, com a F\&A's do P1 apresentando evidências de aumento em duas variáveis (MEBIT e EBITDA), ao passo que as F\&A's ocorridas no $\mathrm{P} 2$ apresentaram evidências apenas para a variável EBITDA (rejeitou-se $\mathrm{H}_{4}$ ) e 5) constatou-se correlação significante ao se compara o comportamento de cada uma das três variáveis de criação de valor nos respectivos períodos, anteriores e posteriores, a um nível de significância de 1\%, ressaltando-se, entretanto, que se observou uma proximidade de comportamen- to, em ordem decrescente, para as variáveis: QT e VM/VP (acima de 84\%), QT e FV (acima de 58\%) e VM/VP e FV (acima de $28 \%$ apenas), sinalizando que a variável VM/VP pode ser utilizada como proxy do QT, que o FV deve sê-lo com ressalvas (baixa correlação), enquanto as variáveis VM/VP e FV não devem ser utilizadas como proxy uma da outra, tendo em vista a baixa correlação apresentadas entre ambas (rejeitou-se $\mathrm{H}_{5}$ ).

Em síntese, por se tratar de uma estratégia complexa, os seus resultados, obtidos sobre processos de F\&A's de empresas brasileiras, têm que ser analisados sob diversos pontos de vista. Entretanto, aparentemente, as conclusões a que se chegou nesta pesquisa vão na direção de que tal estratégia proporciona sinergias operacionais para as empresas, leva à maximização do valor de mercado das empresas pesquisadas e, aparentemente, da riqueza dos acionistas.

Em se tratando de economia brasileira, na literatura nacional consultada, não se encontrou estudo que tivesse analisado amostra tão representativa como a que se empreendeu neste artigo. Além disso, outros aspectos desta pesquisa devem ser destacados como forma de justificá-la e reafirmar suas conclusões, tais como o grupo de variáveis utilizadas e o período analisado, de nove anos, dividido em dois, com características distintas, englobando grandes transformações da economia, reforçando a necessidade de estudos que possibilitem um melhor entendimento do resultado de uma estratégia corporativa tão importante e impactante para as empresas como é uma F\&A.

Cabe salientar que o escopo desta pesquisa não foi

Tabela 3 - Resumo da correlação das variáveis de criação de valor

\begin{tabular}{|c|c|c|c|c|c|c|c|}
\hline \multicolumn{2}{|r|}{$(N=600)$} & QT_A & QT_D & VM/VP_A & VM/VP_D & FV_A & FV_D \\
\hline QT_A & $\begin{array}{l}\text { Pearson Correlation } \\
\text { Sig. (2-tailed) }\end{array}$ & 1 & & & & & \\
\hline \multirow{2}{*}{ QT_D } & Pearson Correlation &, $587(* *)$ & & & & & \\
\hline & Sig. (2-tailed) & ,000 & & & & & \\
\hline VM/VP_A & $\begin{array}{l}\text { Pearson Correlation } \\
\text { Sig. (2-tailed) }\end{array}$ & $\begin{array}{l}, 907(* *) \\
, 000\end{array}$ & $\begin{array}{l}, 611(* *) \\
, 000\end{array}$ & & & & \\
\hline \multirow{2}{*}{ VM/VP_D } & Pearson Correlation &, $236(* *)$ &, $847(* *)$ &, $274(* *)$ & & & \\
\hline & Sig. (2-tailed) & ,000 & ,000 & ,000 & & & \\
\hline FV_A & $\begin{array}{l}\text { Pearson Correlation } \\
\text { Sig. (2-tailed) }\end{array}$ & $\begin{array}{l}, 588(* *) \\
, 000\end{array}$ & $\begin{array}{l}, 374(* *) \\
, 000\end{array}$ & $\begin{array}{l}, 541(* *) \\
, 000\end{array}$ & $\begin{array}{l}, 140(* *) \\
, 001\end{array}$ & & \\
\hline \multirow{2}{*}{ FV_D } & Pearson Correlation &, $771(* *)$ &, $706(* *)$ &, $815(* *)$ &, $280(* *)$ &, $533(* *)$ & \\
\hline & Sig. (2-tailed) & ,000 &, 000 & ,000 &, 000 &, 000 & \\
\hline
\end{tabular}

(**) Correlação com significância ao nível de 0,01 (2-caudas). 
colocar um ponto final na controvérsia existente na literatura sobre o resultado dos processos de F\&A's na perspectiva de criação de valor para o acionista e melhorias do desempenho operacional. Controvérsia esta salutar e instigante do ponto de vista da investigação científica, pois os embates teóricos e evidências empíricas favoráveis e desfavoráveis contribuem sobremaneira para o avanço da ciência e do entendimento mais aprofundado da atividade de F\&A's.

Apesar de relevantes, as evidências encontradas neste estudo devem ser vistas com cautela, principalmente com relação a dois pontos principais. Primeiro, porque os resultados se referem às empresas pesquisadas, a um momento específico da economia brasileira, mas não deixam de ser um indicador do resultado, longe de querer fazer generalizações. O segundo decorre do curto espaço de tempo analisado após a F\&A, cujos desdobramentos podem ainda não ter se refletido totalmente nas operações das empresas. Sobre esse ponto, entretanto, cabe ressaltar que a análise de um período superior a dois anos após a F\&A certamente contemplaria outros eventos e estratégias corporativos importantes, inclusive a realização de outras F\&A's, os quais, certamente poderiam distorcer os resultados.

Cabe ressaltar, por fim, que esses fatores são limitadores do escopo da pesquisa, mas não inviabilizadores dos seus

Tabela 4 - Resumo do resultado do teste de comparação de médias para variáveis de sinergias operacionais

\begin{tabular}{|c|c|c|c|c|c|c|c|c|c|c|}
\hline $\mathrm{N}^{0}$ & AMOSTRA & VARIÁVEL & $\begin{array}{l}\mathrm{N}^{0} \\
\text { OBS. }\end{array}$ & $\begin{array}{l}\text { MÉDIA } \\
\text { ANTES }\end{array}$ & $\begin{array}{l}\text { MÉDIA } \\
\text { DEPOIS }\end{array}$ & $\begin{array}{c}\text { VARIAÇÃO } \\
\text { NA } \\
\text { MÉDIA }\end{array}$ & $\begin{array}{l}\text { CRESCI- } \\
\text { MENTO } \\
\text { NA } \\
\text { VARIÁVEL }\end{array}$ & RESULTADO & $\begin{array}{c}\text { NIIVEL } \\
\text { SIGNIFICÂNCIA } \\
\text { - TESTE } \\
\text { WILCOXON - }\end{array}$ & $\begin{array}{l}\text { NIVEL SIGNI- } \\
\text { FICÂNCIA } \\
\text { - TESTE DO } \\
\text { SINAL- }\end{array}$ \\
\hline 1 & \multirow{4}{*}{$\begin{array}{l}\text { Todas as } \\
\text { Empresas } \\
(\mathrm{N}=76)\end{array}$} & MEBIT & 568 & $13,41 \%$ & $14,31 \%$ & $6,7 \%$ & $56,0 \%$ & melhorou & 0,001 & 0,003 \\
\hline 2 & & EBTIDA & 150 & $\$ 506.829 .751$ & $\$ 756.846 .879$ & $49,3 \%$ & $66,7 \%$ & melhorou & 0,000 & 0,000 \\
\hline 3 & & GBC & 150 & $72,30 \%$ & $75,93 \%$ & $5,0 \%$ & $54,0 \%$ & melhorou & 0,786 & 0,369 \\
\hline 4 & & CRESC. & 592 & $17,7 \%$ & $9,3 \%$ & $-47,2 \%$ & $44,6 \%$ & piorou & 0,001 & 0,010 \\
\hline 1 & \multirow{4}{*}{$\begin{array}{l}\text { Adquirentes } \\
(N=63)\end{array}$} & MEBIT & 480 & $14,24 \%$ & $14,78 \%$ & $3,8 \%$ & $55,0 \%$ & melhorou & 0,013 & 0,022 \\
\hline 2 & & EBTIDA & 126 & $\$ 591.166 .324$ & $\$ 869.659 .849$ & $47,1 \%$ & $65,9 \%$ & melhorou & 0,000 & 0,001 \\
\hline 3 & & GBC & 126 & $83,31 \%$ & $78,77 \%$ & $-5,5 \%$ & $54,8 \%$ & piorou & 0,987 & 0,327 \\
\hline 4 & & CRESC. & 504 & $13,5 \%$ & $7,8 \%$ & $-42,3 \%$ & $45,6 \%$ & piorou & 0,004 & 0,055 \\
\hline 1 & \multirow{4}{*}{$\begin{array}{l}\text { Adquiridas } \\
(\mathrm{N}=13)\end{array}$} & MEBIT & 96 & $8,15 \%$ & $11,27 \%$ & $38,3 \%$ & $64,6 \%$ & melhorou & 0,001 & 0,006 \\
\hline 2 & & EBTIDA & 26 & $\$ 57.529 .451$ & $\$ 149.558 .077$ & $160,0 \%$ & $69,2 \%$ & melhorou & 0,112 & 0,078 \\
\hline 3 & & GBC & 26 & $12,67 \%$ & $55,69 \%$ & $339,5 \%$ & $50,0 \%$ & melhorou & 0,657 & 1,000 \\
\hline 5 & & CRESC. & 96 & $38,2 \%$ & $17,3 \%$ & $-54,6 \%$ & $70,8 \%$ & piorou & 0,108 & 0,052 \\
\hline 1 & \multirow{4}{*}{$\begin{array}{l}\text { Fusões } \\
(\mathrm{N}=6)\end{array}$} & MEBIT & 48 & $14,23 \%$ & $16,14 \%$ & $13,4 \%$ & $75,0 \%$ & melhorou & 0,008 & 0,001 \\
\hline 2 & & EBTIDA & 12 & $\$ 313.270 .917$ & $\$ 506.995 .333$ & $61,8 \%$ & $66,7 \%$ & melhorou & 0,272 & 0,388 \\
\hline 3 & & GBC & 12 & $88,52 \%$ & $46,36 \%$ & $-47,6 \%$ & $33,3 \%$ & piorou & 0,084 & $0,388(a)$ \\
\hline 4 & & CRESC. & 48 & $3,1 \%$ & $30,6 \%$ & $892,3 \%$ & $52,1 \%$ & melhorou & 0,594 & 0,885 \\
\hline 1 & \multirow{4}{*}{$\begin{array}{l}\text { Período } 1 \\
(\mathrm{~N}=36)\end{array}$} & MEBIT & 256 & $5,01 \%$ & $6,46 \%$ & $28,9 \%$ & $63,3 \%$ & melhorou & 0,000 & 0,000 \\
\hline 2 & & EBTIDA & 70 & $\$ 146.506 .608$ & $\$ 232.013 .427$ & $58,4 \%$ & $67,1 \%$ & melhorou & 0,008 & 0,006 \\
\hline 3 & & GBC & 70 & $46,33 \%$ & $60,69 \%$ & $31,0 \%$ & $55,7 \%$ & melhorou & 0,617 & 0,403 \\
\hline 5 & & CRESC. & 272 & $25,23 \%$ & $12,32 \%$ & $-51,2 \%$ & $43,0 \%$ & piorou & 0,007 & 0,025 \\
\hline 1 & \multirow{4}{*}{$\begin{array}{l}\text { Período } 2 \\
(\mathrm{~N}=40)\end{array}$} & MEBIT & 312 & $20,31 \%$ & $20,75 \%$ & $2,1 \%$ & $50,0 \%$ & melhorou & 0,319 & 0,955 \\
\hline 2 & & EBTIDA & 80 & $\$ 822.112 .500$ & $\$ 1.216 .076 .150$ & $47,9 \%$ & $66,3 \%$ & melhorou & 0,003 & 0,005 \\
\hline 3 & & GBC & 80 & $95,02 \%$ & $89,26 \%$ & $-6,1 \%$ & $52,5 \%$ & piorou & 0,920 & 0,737 \\
\hline 4 & & CRESC. & 320 & $11,3 \%$ & $6,8 \%$ & $-39,7 \%$ & $45,9 \%$ & piorou & 0,030 & 0,162 \\
\hline
\end{tabular}


resultados. Diante das dificuldades inerentes ao tema - e partidário da opinião de que os processos de F\&A's devem ser analisados sob a perspectiva de um mosaico de resultados obtidos com diferentes dados e metodologias, que por si só não explicam o todo, mas em conjunto fornecem um panorama mais amplo desse debate e possibilitam um melhor entendimento do assunto - este trabalho deu sua contribuição ao fornecer evidências de que os processos de F\&A's das empresas brasileiras analisadas aparentemente beneficiaram os acionistas e resultaram na geração de sinergias operacionais, confirmando-se a hipótese da eficiência apresentada por Weston (1994).
Como sugestão para pesquisas futuras, recomenda-se que se proceda a uma análise estratégica, conciliando metodologias quantitativas e qualitativas, de maneira a melhor entender os resultados das F\&A's.

\section{REFERÊNCIAS}

AGRAWAL, A; JAFFE, J; MANDELKER, G. The post-merger performance of acquiring firms: a reexamination of an anormaly. The Journal of Finance, v. 47, n. 4 , p. 1605-1621, 1992.

\section{Quadro 4 - Resumo dos resultados encontrados na pesquisa}

\section{AMOSTRA \\ VARIÁVEIS \\ HIOPÓTESE(S) \\ RESULTADO / CONCLUSÃO}

Todas as
Empresas
$(\mathrm{N}=76)$

Adquirentes,

Adquiridas e

Fusões

$(\mathrm{N}=36)$

Período 2

$(\mathrm{N}=40)$
MEBIT, EBTIDA, GBC, CRESC.

QT

VM/VP

FV

QT

VM/VP

FV

$\mathrm{H}_{2} \mathrm{e} \mathrm{H}_{5}$ rejeitadas

$\mathrm{H}_{2}$

MEBIT, EBTIDA, rejeitada, mas

GBC, CRESC. com resultados contraditórios

$\mathrm{H}_{3}$ rejeitada

$\mathrm{FV}$

MEBIT, EBTIDA, GBC, CRESC.

$\mathrm{H}_{3}$ rejeitada

QT

VM/VP

FV

$\mathrm{H}_{4}$ rejeitada

$\mathrm{H}_{4}$ rejeitada, mas com resultados contraditórios

$\mathrm{H}_{4}$ rejeitada

$\mathrm{H}_{4}$ MEBIT, EBTIDA, GBC, CRESC.
F\&A's estudadas resultaram na maximização do valor de mercado das empresas pesquisadas, e conseqüentemente, da riqueza dos acionistas. Existe correlação significante entre essas três variáveis, podendo ser utilizadas como proxies alternativas umas das outras em estudos acadêmicos.

F\&A's estudadas levaram a uma melhora no desempenho operacional das empresas após a F\&A (exceto a variável Cres.), ou seja, resultaram em sinergias operacionais.

Empresas adquirentes apresentaram evidências de maior potencial de criação de valor após a união, quando comparadas com empresas adquiridas e fusionadas.

Empresas adquirentes apresentaram maior potencial de gerar sinergias operacionais, quando comparadas com empresas adquiridas e fusionadas (evidências de melhoras na MEBIT e EBITDA), enquanto que as empresas adquiridas também o tiveram, mas somente na última variável.

F\&A's apresentaram apenas indícios de melhora (sem significância estatística) nas variáveis de criação de valor.

Resultados contrários aos esperados. F\&A's apresentaram melhoras na MEBIT, EBITDA e GBC, com significância estatística nas duas primeiras.

Apresentaram evidências (significância estatística) de melhoras nas três variáveis de criação de valor.

F\&A's apresentaram melhoras apenas nas duas primeiras variáveis e com significância estatística apenas para a variável EBITDA. F\&A's do P1 apresentaram um melhor desempenho operacional do que as do P2. 
ANDRADE, G; MITCHELL, M; STAFFORD, E. New evidence and perspectives on mergers. Journal of Economic Perspectives, v. 15, n. 1, 2001, p. 103-120, 2001.

ASQUITH, P. Merger bids, uncertainly and stockholder returns. Journal of Financial Economics, v. 11, n. 1-4, p. 51-83, 1983.

ASSAF NETO, A. Contribuição ao estudo de avaliação de empresas no Brasil: uma aplicação prática. 2003. 203 f. Tese de Livre-Docência, Faculdade de Economia, Administração e Contabilidade, Universidade de São Paulo, Ribeirão Preto, 2003.

BARNEY, J. Return to bidding firms in mergers and acquisitions: reconsidering the relatedness hypothesis. Strategic Management Journal, v. 9 , Special Issue, p. 71-78, 1988.

BERLE JR., A. A; MEANS, G. C. The modern corporation and private propriety. New York: Macmillan, 1932.

CAMARGOS, M. A; BARBOSA, F. V. Análise do desempenho econômicofinanceiro e da criação de sinergias em processos de fusões e aquisições do mercado brasileiro ocorridos entre 1995 e 1999. Caderno de Pesquisas em Administração USP, v. 12, n. 2, p. 99-115, 2005.

CANO, M. O recente processo de fusões e aquisições na economia brasileira. 164 f. Dissertação de Mestrado em Economia, Instituto de Economia, Universidade Estadual de Campinas, Campinas, 2002.

CAPRON, L; PISTRE, N. When do acquirers earn abnormal returns? Strategic Management Journal, v. 23, n. 9, p. 781-94, 2002.

CHATTERJEE, S. Types of synergy and economic value: the impact of acquisitions on merging and rival firms. Strategic Management Journal, v. 7 , n. 2, p. 119-39, 1986

CHATTERJEE, S. Sources of value in takeovers: synergy or reestructuring - implications for target and bidder firms. Strategic Management Journal, v. 13, n. 4 , p. 267-86, 1992.

CHUNG, K; PRUITT, S. A simple approximation of Tobin's Q. Financial Management, v. 23, n. 3, p. 70-74, 1994.

COOPER, D. R; SCHINDLER, P. S. Métodos de pesquisa em administração. 7. ed. Porto Alegre: Bookman, 2003. 640 p.

DAMODARAN, A. Finanças corporativas: teoria e prática. 2. ed. Porto Alegre: Bookman, 2004. 796 p.

FAMÁ, R; BARROS, L. A. Q de Tobin e seu uso em finanças: aspectos metodológicos e conceituais. Caderno de Pesquisas Administração USP, v. 7 , n. 4 , p. $27-43,2000$
GOMES, C; AIDAR. O; VIDEIRA, R. Fusões, aquisições e lucratividade: uma análise do setor siderúrgico brasileiro. Economia, v. 7, n. 4, p.143163, 2006.

GRAHAM, J. R; LEMMON, M. L; WOLF, J. G. Does corporate diversification destroy value? The Journal of Finance, v. 57, n. 2, 695-720, 2002.

HASBROUCK, J. The characteristics of takeover targets: $Q$ and other measures. Journal of Banking and Finance, v. 9, n. 3, p. 351-362, 1985.

HEALY, P; PALEPU, K; RUBACK, R. Does corporate performance improve after mergers? Journal of Financial Economics, v. 31, v. 2, p. 135176,1992

HELFERT, E. A. Técnicas de análise financeira. 9. ed. Porto Alegre: Bookman, 2000.

JENSEN, M. C; RUBACK, R. S. The market for corporate control: the scientific evidence. Journal of Financial Economics, v. 11, n. 1-4, p. 5-50, 1983.

KLOECKNER, G. O. Fusões e aquisições: motivos e evidência empírica. Revista de Administração, São Paulo, v. 29, n. 1, p. 42-58, 1994.

LANG. L; STULZ, R. M. Tobin's Q, corporate diversification and firm performance. Journal of Political Economy, v. 102, n. 6, p. 1248-1280, 1994

LANG, L; STULZ, R; WALKLING, R. Tobin's Q and the gains from successful tender offers. Jounal of Financial Economics, v. 24, n. 3, p. 137154,1989

LLOYD, W. P; JAHERA, J. S. J. Firm-diversification effects on performance as measured by Tobin's Q. Managerial and Decision Economics, v. 15, n 3, p. 259-266, 1994.

LOUGHRAN, T; VIJH, A. Do long-term shareholders beneft from corporate acquisitions? Unpublished paper. Apr. 1997.

MEGGINSON, W. L; NASH, R. C; RANDENBORGH, M. The financial and operating performance of newly privatized firms: an international empirical analysis. The Journal of Finance, v. 49, n. 2, p. 391-9, 1994.

MATIAS, A. B; PASIN, R. M. A geração de sinergias e seus impactos na rentabilidade das empresas nos casos de fusões e aquisições. Revista de Administração, São Paulo, v. 36 n. 1, p. 5-13, 2001.

MCGEE, R. W. The economics of mergers and acquisitions. The Mid-Atlantic Journal of Business, v. 25, n. 4, p. 45-55, 1989.

NAPIER, N. K. Mergers and acquisitions, human resources issues and outcomes: a review and suggested typology. Journal of Management Studies, v. 26, n. 3, p. 271-286, 1989. 
PASIN, R. M. Fusões e aquisições na indústria de alimentos do Brasil: um estudo sobre a gestão financeira das empresas. Anais Eletrônicos. Flórida: BALAS, 2002. (CD-ROM).

PINHEIRO, A. C. Impactos microeconômicos da privatização no Brasil. Pesquisa e Planejamento Econômico, v. 26, n. 3, p. 357-398, 1996.

RAVENSCRAFT, D. J; SCHERER, F. M. Mergers, selloffs, and economic efficiency. Washington: Brookings Institution, 1987.

ROCHA, F; IOOTTY, M; FERRAZ, J. C. Desempenho das fusões e aquisições na indústria brasileira na década de 90: a ótica das empresas adquiridas. Revista de Economia Contemporânea, v. 5, n. especial, p. 69-102, 2001

SALTER, M. S; WEINHOLD, W. A. Diversification through acquisition: strategies for creating economic value. New York: The Free Press, 1979.

SILVA, J. P. Análise financeira das empresas. 8. ed. São Paulo: Atlas, 2006 $552 \mathrm{p}$.
SILVEIRA, A. D. M. da. Governança corporativa e estrutura de propriedade: determinantes e relação com o desempenho das empresas no Brasil. 2004 250 f. Tese de Doutorado em Administração, Programa de Pós-Graduação em Administração, Universidade de São Paulo, São Paulo, 2004.

STEINER, T. L. A reexamination of the relationships between ownership structure, firm diversification, and Tobin's Q. Quarterly Journal of Business and Economics, v. 35, n. 4, p. 39-48,1996.

TOBIN, J; BRAINARD, W. Pitfalls in financial models building. American Economic Review, v. 58, n. 2, 1968.

TRAVLOS, N. G. Corporate takeover bids, methods of payment, and bidding firms' stock returns. The Journal of Finance, v. 42, n. 4, p. 943-63, 1987.

WESTON, J. F. The payoff in merger and acquisitions. In: ROCK, M; ROCK, R. H; SIKORA, M (Eds) The MEA Handbook. $2^{\text {nd }}$ ed. New York: McGraw-Hill, 1994.

WERNERFELT, B; MONTGOMERY, C. A. Tobin's Q and the importance of focus in firm performance. American Economic Review, v. 78, n. 1, p. 246-250, 1988 